Smoothed Emission for IMC

N. A. Gentile

February 7, 2011 
This document was prepared as an account of work sponsored by an agency of the United States government. Neither the United States government nor Lawrence Livermore National Security, LLC, nor any of their employees makes any warranty, expressed or implied, or assumes any legal liability or responsibility for the accuracy, completeness, or usefulness of any information, apparatus, product, or process disclosed, or represents that its use would not infringe privately owned rights. Reference herein to any specific commercial product, process, or service by trade name, trademark, manufacturer, or otherwise does not necessarily constitute or imply its endorsement, recommendation, or favoring by the United States government or Lawrence Livermore National Security, LLC. The views and opinions of authors expressed herein do not necessarily state or reflect those of the United States government or Lawrence Livermore National Security, LLC, and shall not be used for advertising or product endorsement purposes.

This work performed under the auspices of the U.S. Department of Energy by Lawrence Livermore National Laboratory under Contract DE-AC52-07NA27344. 


\title{
Smoothed Emission for IMC
}

\author{
N. A. Gentile
}

Lawrence Livermore National Laboratory

L-38 P. O. Box 808 Livermore, CA 94550 gentile1@llnl.gov 


\section{The current source algorithm for IMC}

Here is a review of the current way we handle source photons in IMC.

A source photon is created with a randomly sampled position $x_{p}$ in the zone, a direction $\Omega_{p}$, a frequency $v_{p}$ sampled from the appropriate distribution, and a time $t_{p}$ uniformly sampled from $\left[t^{n}, t^{n+1}\right]$. The source photons each have an energy $E_{p}$. The sum of $E_{p}$ over all of the photons equals the energy of the source for that time step. In the case of thermal emission in a zone with volume $V$, we would have

$$
\sum_{p=1}^{N} E_{p}=\sigma_{P} a c T^{4} V \Delta t,
$$

where $N$ is the number of thermal source photons for that time step, and $\sigma_{P}$ is the Planck mean opacity.

Census photons do not differ from source photons in any way, except that they all start the time step with $t_{p}=t^{n}$.

Then we advance each photon until it reaches the end of the time step. This advance consists of 4 steps.

Step 1 of this process is to calculate the following quantities: the distance to the zone boundary $d_{b}\left(x_{p}, \Omega_{p}\right)$; the distance to scatter $d_{s}=-\ln (r) / \sigma_{s}$, where $\mathrm{r}$ is a randon number uniform in $[0,1]$; and the distance to census $d_{c}=t^{n+1}-t_{p}$. We take the minimum of these 3 distances: $d_{p}=\operatorname{Min}\left(d_{b}, d_{s}, d_{c}\right)$.

Step 2 is to advance the photon a distance $d_{p}$. We change the photon's position $x_{p}->$ $x_{p}+\Omega_{p} d_{p}$, the photon time to $t_{p}+d_{p} / c$, and remove an amount of energy $E_{p}\left[1-\exp \left(-\sigma_{a} d_{p}\right)\right]$ from the photon. This energy is added into the material, because it represents aborption.

Step 3 is to simulate the physical activity that was supposed to occur at the distance $d_{p}$. For example, if $d_{p}=d_{s}$, we would do a scatter, which would result in a change of $\Omega_{p}$. If $d_{p}=d_{b}$, we move the photon to a new zone. If $d_{p}=d_{c}$, we are through advancing the photon in this time step.

Step 4 is to go back to step 1 , unless the photon we are advancing has reached census. Since we keep increasing $t_{p}$, the photon will eventually reach census, and the proccess will terminate.

When we are done with all of the photons, we update the matter temperature using the difference between the emmitted and absorbed energy, and proceed to the next time step.

Let's look at applying this process to one time step of a problem in thermal equilibrium, where the matter temperature $T_{m}=$ the radiation temperature $T_{r}=T_{0}$. To simplify things, we'll have one zone with volume $V$ and reflecting boundaries, so that we are simulating an infinite medium. To further simplify, we will assume that $\sigma$ is independednt of frequency.

We start the simulation with an initial set of $N_{c}$ photons, each with $t_{p}=0$ and initial energies satisfying

$$
\sum_{p=1}^{N_{c}} E_{p}(t=0)=a T_{0}^{4} V .
$$

These photons represent the initial radiation energy in the problem. Each of these photons will be advanced in time from $t=0$ to $t=\Delta t$, and each will travel a distance $c \Delta t$. So each photon will reach $\Delta t$ with an energy $E_{p}(t=\Delta t)=E_{p}(t=0) \exp (-\sigma c \Delta t)$. So the radiation energy density due to the census photons at the end of the time step will be

$$
E_{r, \text { census }}=\sum_{p=1}^{N_{c}} E_{p}(t=\Delta t)
$$




$$
\begin{aligned}
& =\sum_{p=1}^{N_{c}} E_{p}(t=0) \exp (-\sigma c \Delta t) \\
& =a T_{0}^{4} V \exp (-\sigma c \Delta t) .
\end{aligned}
$$

To simulate thermal emission, we will make $N_{s}$ thermal source photons, each with a different initial time $t_{i, p}$, with energies that satisfy

$$
\sum_{p=1}^{N_{s}} E_{p}(t=0)=a c \sigma_{P} T_{0}^{4} V \Delta t
$$

We will make the energy of all the thermal source particles equal, so for these particles $E_{p}(t=$ $t_{i, p}=a c \sigma_{P} T_{0}^{4} V \Delta t / N_{s}$.

Each thermal source photon will move from the time it is created until the end of the time step. Since $t_{i, p}$ is different for each thermal source photon, they will all reach time $\Delta t$ with different energies $E_{p}(t=\Delta t)=E_{p}(t=0) \exp \left[-\sigma c\left(\Delta t-t_{i, p}\right)\right]$

The sum of these energies will be

$$
\begin{aligned}
\sum_{p=1}^{N_{s}} E_{p}(t=\Delta t) & =\sum_{p=1}^{N_{s}} E_{p}(t=0) \exp \left[-\sigma c\left(\Delta t-t_{i, p}\right)\right] \\
& =a T_{0}^{4} V c \sigma \Delta t \frac{1}{N_{s}} \sum_{p=1}^{N_{s}} \exp \left[-\sigma c\left(\Delta t-t_{i, p}\right)\right]
\end{aligned}
$$

where we have used the assumption that all thermal photons had the same initial energy.

In the limit $N_{s} \rightarrow \infty$,

$$
\frac{1}{N_{s}} \sum_{p=1}^{N_{s}} \exp \left[-\sigma c\left(\Delta t-t_{i, p}\right)\right] \rightarrow \frac{1}{\Delta t} \int_{0}^{\Delta t} \exp [-\sigma c(\Delta t-\tau] \mathrm{d} \tau .
$$

In other words, the sum in Eq.(5) is a Monte Carlo estimate for an integral over all possible thermal emission times. The integral in Eq.(6) can be performed analytically:

$$
\int_{0}^{\Delta t} \exp \left[-\sigma c(\Delta t-\tau] \mathrm{d} \tau=\frac{1-\exp [-c \sigma \Delta t]}{c \sigma} .\right.
$$

Using this expression, we find that, in the limit of a large number of particles, the radiation energy due to thermally emitted photons at $t=\Delta t$ will be

$$
a E_{r, \text { thermal }}=a T_{0}^{4} V(1-\exp [-c \sigma \Delta t]),
$$

and the sum of $E_{r, c e n s u s}$ given in Eq.(3), and $E_{r, \text { thermal }}$ will be $a T_{0}^{4} V$, which is the value necessary to maintain thermal equilibrium. The matter energy will also be the same as the initial value, by energy conservation.

With a finite number of photons, we will not maintain thermal equilibrium, because the sum in Eq.(5) will only approximate the integral.

The important point is that, for this infinite medium problem, even with a finite number of particles, the census photons will give exactly the right value for their contribution to the radiation 
energy, but the thermally emitted photons will not. This happens because the emission is sampled at discrete times, so the contribution to the radiation energy density at the end of the time step from the thermal photons is only approximate.

This is illustrated in Fig. 1. This plot shows an IMC simulation using one zone, a cube with unit length in each direction. All faces have reflecting boundaries, making it effectively an infinite medium problem. Thr material and radiation temperatures were initialized to 1 . The material has a heat capacity $c_{v}=1.0$, and an absorption opacity $\sigma=10$. The simulation used 100 photons per time step, and units were chosen so that $a=c=1$. The simulation used $\Delta t=0.001$ from $t=0$ to $t=1, \Delta t=0.01$ from $t=1$ to $t=2$, and $\Delta t=0.1$ for $t>2$.

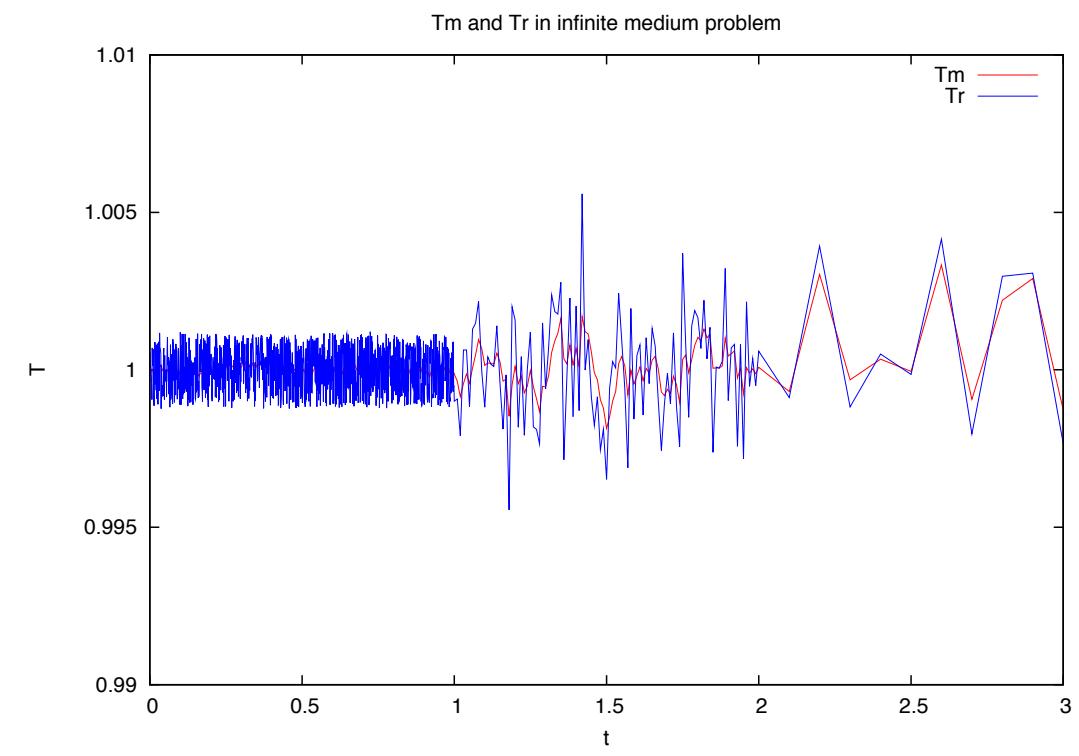

Figure 1: Matter and radiation temperature for infinite medium test problem using IMC with three different time steps

Fig. 1 shows the statistical noise in the IMC simulation. The noise increases with $\Delta t$. This happens because the integrand in Eq.(6) changes value more when $\Delta t$ is large - the exponential decay of the energy of the thermal photons is larger over a larger time step. When the integrand varies more, a Monte Carlo approximation, like the sum in Eq.(6) has a larger variance, so the statistical noise increases.

As noted above, the noise in the simulation is caused by the choice of emission times for the thermally emitted photons. If we could eliminate the statistical noise caused by the choice of the emission time, then the matter and radiation temperature in Fig. 1 would be exactly 1 at all times, independent of $\Delta t$.

This is illustrated further in Figs. 2 and 3. These plots show the results of simulations that are the same as that in Fig. 1, except that the problem does not begin in equilibrium and $\Delta t$ is 
fixed at 0.001 .

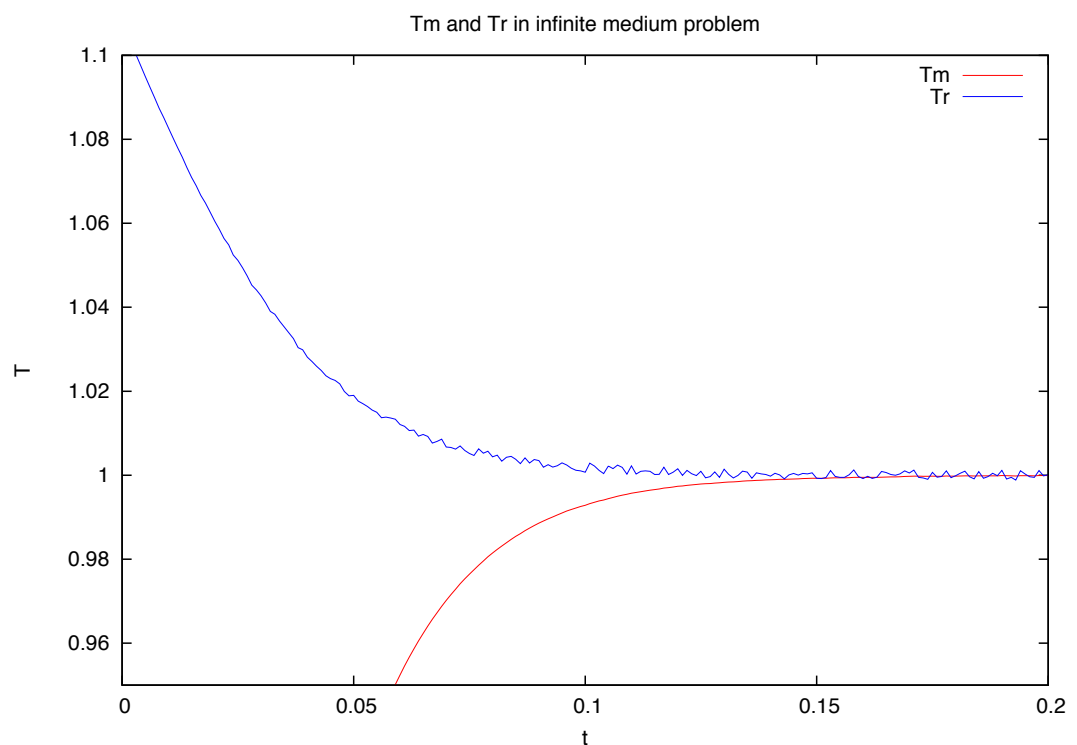

Figure 2: Matter and radiation temperature using IMC for infinite medium test problem with $T_{r}>T_{m}$ initially.

In the simulation in Fig. 2, the initial radiation temperature was higher than the initial matter temperature. Because of the low initial matter temperature, there is less thermal emission at the beginning of the problem than at late times, and the radiation energy density at early times is dominated by the initial census photons. As $T_{m}$ becomes closer to $T_{r}$, photons created by thermal emission become more important. Fig. 2 shows that the radiation temperature is less noisy at early times, and becomes noisy when $T_{m}$ becomes closer to $T_{r}$.

In Fig. 3, we show the results of a simulation in which $T_{m}$ is initially much larger than $T_{r}$. In this simulation, thermally emitted photons are always important, and the radiation temperature shows statistical noise even at early times.

The results of the simulations shown in Figs. 2 and 3 are consistent with the idea that the thermal photons, because of the statitical noise created by the selection of their emission times, are the source of statistical noise in infinite medium Monte Carlo simulations.

We have only considered infinite medium problems in this section. In a simulation with multiple zones, we would also see statistical noise from the random choice of position and direction of both the thermally emitted photons and the census photons. So eliminating the noise from the sampling of emission times would not eliminate all statistical noise in more complicated simulations. However, some simulations with opaque zones with small time steps can have zones where most of the thermally emitted photons are absorbed before leaving the zone. These simulations could concievably show a reduction of statistical noise if the sampling of the time of thermal 


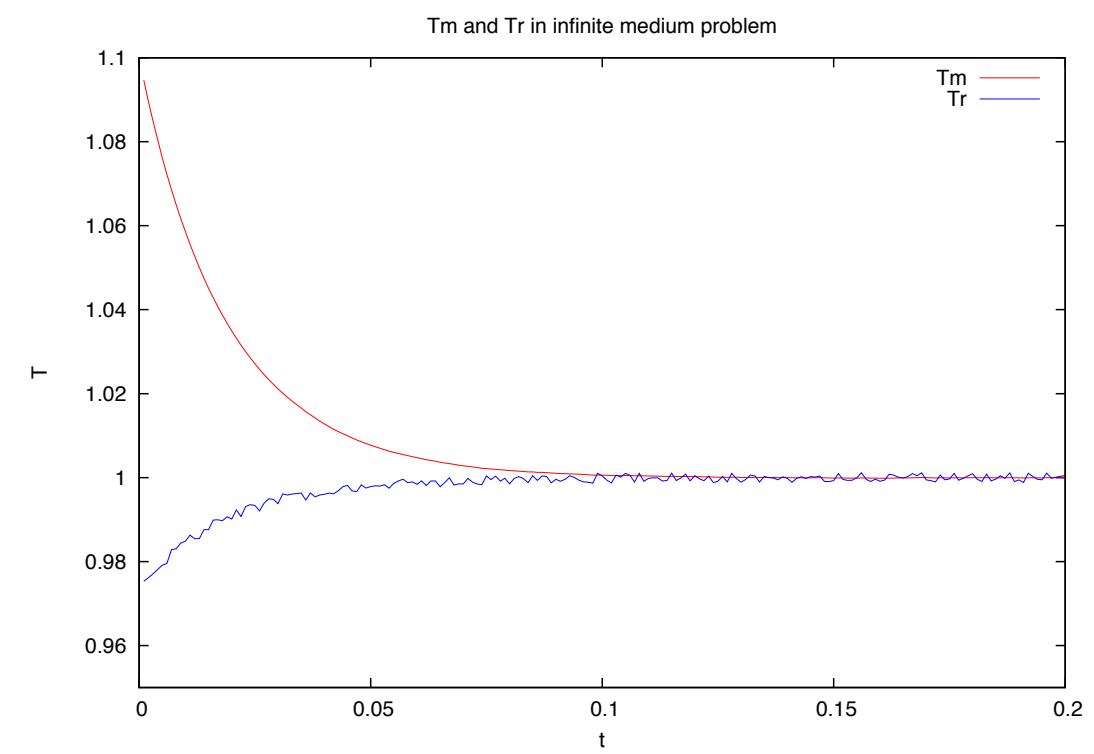

Figure 3: Matter and radiation temperature using IMC for infinite medium test problem with $T_{m}>T_{r}$ initially.

emission was made more accurate.

In the next section, we will discuss a way to eliminate the statistical noise due to the sampling of emission time. 


\section{The speculative new source algorithm for IMC}

In the last section, we established that the statistical noise due to the choice of the emission time of thermal source photons caused the noise in the temperature of infinite medium problems. In this section, we will show how to eliminate this noise.

In the IMC algorithm, we regard physical quantities like opacity and heat capacity as constant throughout the time step. This means that quantities like the distance to scatter, and the amount of absorption on a given photon path are independent of the time during the time step. We can take advantage of this fact to calculate the contribution that is made for every emission time between $t^{n}$ and $t^{n+1}$ for each path taken by a source photon. We will refer to this as the modified IMC algorithm in the remainder of this document. Census photons, which have a definite time (specifically, the begining of the time step, $t^{n}$ ), are treated in exactly the same as in standard IMC.

The modified IMC algorithm is best illustrated by describing how we simulate the behavior of a source photon. As in the standard IMC algorithm, we sample a position $x_{p}$, a direction $\Omega_{p}$ , and a frequency $v_{p}$. The energy of the source photon, $E_{p}$ is also sampled in the same way as standard IMC. However, we do not sample an emission time for the photon. Instead, we will regard the energy as being "spread out" over the whole time step, from $t^{n}$ to $t^{n+1}$. The energy emitted in a small period $\left[t_{e}, t_{e}+d t\right]$ will be $d E=\frac{E_{p}}{\Delta t} d t$. So in the modified IMC algorithm, we can think of a source photon with a definite emission time and energy $E_{p}$ as being replaced by a large number of small sub-photons, with emission times spread out evenly between $t^{n}$ to $t^{n+1}$, each with energy $d E$.

Instead of the source photon having a time that we update as it travels, we will keep track of how far it travels, which we will denote by $s_{p}$. The initial value of $s_{p}$ is 0 , During the whole time step, it will travel a total distance $c \Delta t$, unless it exits the problem through a boundary.

Since the modified IMC source photon does not have a time, it does not reach census in the traditional way. Some fraction of its energy will reach census on each path, and some fraction will be absorbed. These fractions will be functions of the initial and final value of $s_{p}$ on the path. The total energy $E_{p}(s=0)$ of the source photon will be either absorbed, leave the problem through a boundary, or reach census as the photon reaches $s_{p}=c \Delta t$. That is, $E_{p}\left(s_{p}=c \Delta t\right)=0$.

First, we will calculate the amount of energy that reaches census on a given path. We will characterize the path as extending from $s_{0}$ to $s_{1}=s_{0}+d_{p}$. Since the absorption opacity is constant during the time step, the energy emitted in a small time period $\left[t_{e}, t_{e}+d t\right]$ (which we can think of as one of our sub-photons) will change to $d E\left(s_{0}+d_{p}\right)=d E\left(s_{0}\right)\left[1-\exp \left(-\sigma_{a} d_{p}\right)\right]$, independent of the value of $t_{e}$, on a path of length $d_{p}$. The sub-photons born between $t^{n+1}-s_{0} / c$ and $t^{n+1}-s_{1} / c$ will reach census during the path, and we want to know the sum of their energies.

When the photon has moved a distance $s_{0}$, the total energy that our photon has consists of emission that occured for $t_{e}$ in the range $\left[t^{n}, t^{n+1}-s_{0} / c\right]$. This range has a size of $t^{n+1}-s_{0} / c-t^{n}=$ $\Delta t-s_{0} / c$, so

$$
d E\left(s_{0}\right)=\frac{E_{p}\left(s_{0}\right)}{\left[\Delta t-s_{0} / c\right]}
$$

and

$$
d E(s)=d E\left(s_{0}\right) \exp \left[-\sigma\left(s-s_{0}\right)\right] .
$$

The total energy reaching census is the integral of Eq.(10 over the range $\left[s_{0}, s_{1}\right]$ :

$$
E_{c}\left(s_{0}, s_{1}\right)=\int_{s_{0}}^{s_{1}} d E(s)
$$




$$
\begin{aligned}
& =\frac{E_{p}\left(s_{0}\right)}{\left[\Delta t-s_{0} / c\right]} \int_{s_{0}}^{s_{1}} \exp \left[-\sigma\left(s-s_{0}\right)\right] \\
& =\frac{E_{p}\left(s_{0}\right)}{\sigma\left[\Delta t-s_{0} / c\right]} 1-\exp \left[-\sigma\left(s_{1}-s_{0}\right)\right] .
\end{aligned}
$$

Next, we will calculate the amount of energy that is absorbed on the path from $s_{0}$ to $s_{1}$. This is done by conservation of energy. As thephoton travels from $s_{0}$ to $s_{1}$, the energy it has is either lost to absorption, reaches census, or remains with the photon (in the form of sub-photons that haven't reaced census yet. So the photon energy at $s_{1}$ is related to the photon energy at $s_{0}$ by

$$
E_{a}\left(s_{0}, s_{1}\right)+E_{c}\left(s_{0}, s_{1}\right)+E_{p}\left(s_{1}\right)=E_{p}\left(s_{0}\right) .
$$

We know from Eq.(9) how the energy $d E(s)$ of a sub-photon is related to the energy $E_{p}$ of the photon. We can use that to substitute for $E_{p}\left(s_{1}\right)$ in Eq.(12. This results in

$$
E_{a}\left(s_{0}, s_{1}\right)=E_{p}\left(s_{0}\right)\left(1-\exp \left[-\sigma\left(s_{1}-s_{0}\right)\right]\right) \frac{\Delta t-s_{1} / c}{\Delta t-s_{0} / c}-E_{c}\left(s_{0}, s_{1}\right) .
$$

Although the results in this document show a problem in which the only source is thermal emission, the modified algorithm could be applied to other photon sources, such as face sources.

The advance of a source photon in the modified IMC algorithm consists of 4 steps.

Step 1 of is to calculate the following two quantities: the distance to the zone boundary $d_{b}\left(x_{p}, \Omega_{p}\right)$ and the distance to scatter $d_{s}=-\ln (r) / \sigma_{s}$. We take the minimum of these 2 distances: $d_{p}=\operatorname{Min}\left(d_{b}, d_{s}\right)$.

Step 2 is to advance the photon a distance $d_{p}$. We change the photon's position $x_{p}->$ $x_{p}+\Omega_{p} d_{p}$, and we update the distance the photon has traveled in this time step $s_{p}->s_{p}+d_{p}$. We calculate the amount of energy that reaches census and the amount that is absorbed via Eqs(11) and 13. These values are stored in the appropriate arrays, and the energy is removed from the photon.

Step 3 is to simulate the physical activity that was supposed to occur at the distance $d_{p}$. For example, if $d_{p}=d_{s}$, we would do a scatter, which would result in a change of $\Omega_{p}$. If $d_{p}=d_{b}$, we move the photon to a new zone. Unlike standard IMC, we do not have a census event, because energy reaches census on every path.

Step 4 is to go back to step 1, unless the photon we are advancing has traveled a distance $s_{p}=c \Delta t$. As mentioned previously, this will also cause $E_{p}=0$. Since we keep increasing $s_{p}$, the photon will eventually run out of energy, and the proccess will terminate.

There is one aditional feature of the modified IMC algorithm that has to do with the fact that no photons reach census. We have calculated an amount of energy that reaches census in each zone, through the 4 step proceedure outlied above. In the next time step, we need to represent this energy with photons. We can do this by randomly copying the source photons as we advance them. At the end of the time step, we give those copied photons energies that add up to the energy that reached census in the zone. These photons become census photons for the next time step, and are advanced like regular IMC photons. This is how the python code that implements the modified IMC algorithm

Now we will look at results for the test problems described in the previous section calculated by the modified IMC method. The first test is an infinite medium with initial values $T_{m}=T_{r}=$ 1.0. The IMC results were depicted in Fig. 1. The modified IMC results are show in Fig. 4

Fig. 4 shows that there is no statistical noise in the modified IMC simulation for any value of $\Delta t$. This happens because the source photons in the modified IMC algorithm each contribute 


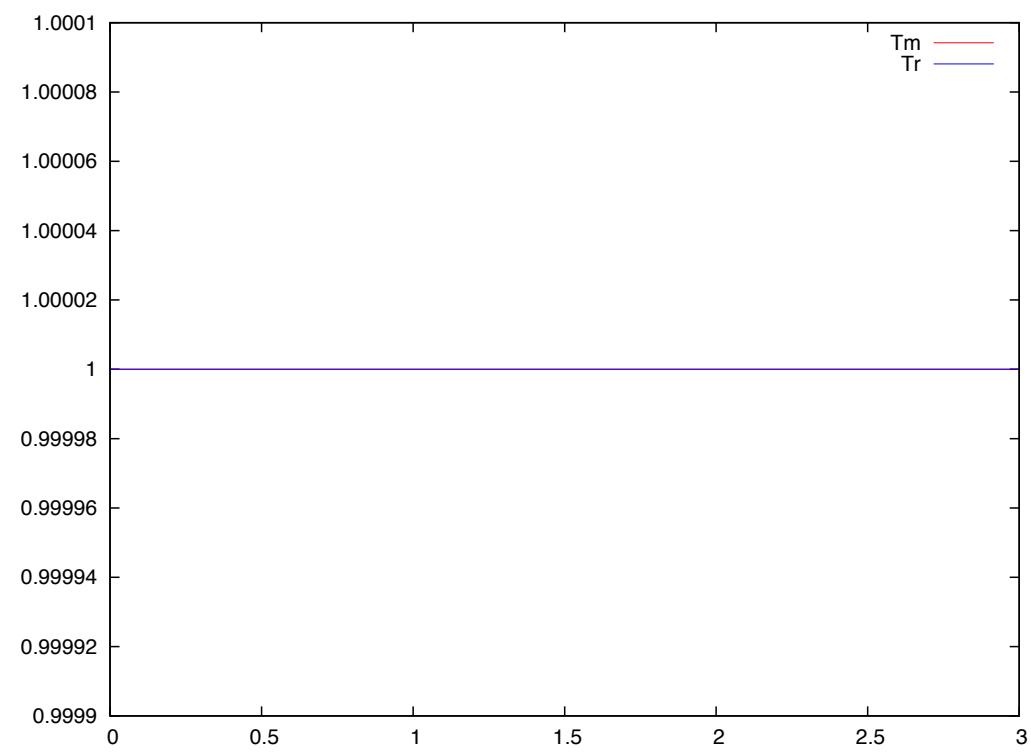

Figure 4: Matter and radiation temperature for infinite medium test problem using modified IMC with three different time steps

exactly the amount of energy calculated in Eq.(5) to census. In effect, the integrand in Eq.(6) is evaluated exactly, not approximately as a sum over a finite number of emission times. The value of $T_{r}$ at the end of the time step is 1.0 to roundoff. By conservation of energy, $T_{m}=1.0$ to roundoff at the end of the time step also, and so these values are maintained throughout the calculation.

Modified IMC results for the problems depicted in Figs. 2 and 3 are shown in Figs. 5 and 6 respectively. Both of these test problems begive out of equilibrium, and in both cases the modified IMC algorithm produces answers with no statistical noise.

The modified IMC algorithm completely eliminates the statistical noise in these infinite medium problems. This happens because the only source of statistical noise in these problems in the emission time. The position and direction of the photons are chosen using random numbers but do not effect the result. I don't expect such spectacular results in multi-zone problems. In those problems, there will be statistical noise from the position and direction, which will show up in how much energy gets depositied by the source photons that cross zone boundaries.

It is possible that the noise from photons the cross zone boundaries will completely swamp the reduction in noise we obtain by using the modified IMC algorithm. My hope is that we will see some reduction in noise in some circumstances. For example, problems with small $\Delta t$ and large $\sigma$ can have reletively few photons that cross zone boundaries. The modified IMC algorithm might show less noisy results on these problems. 


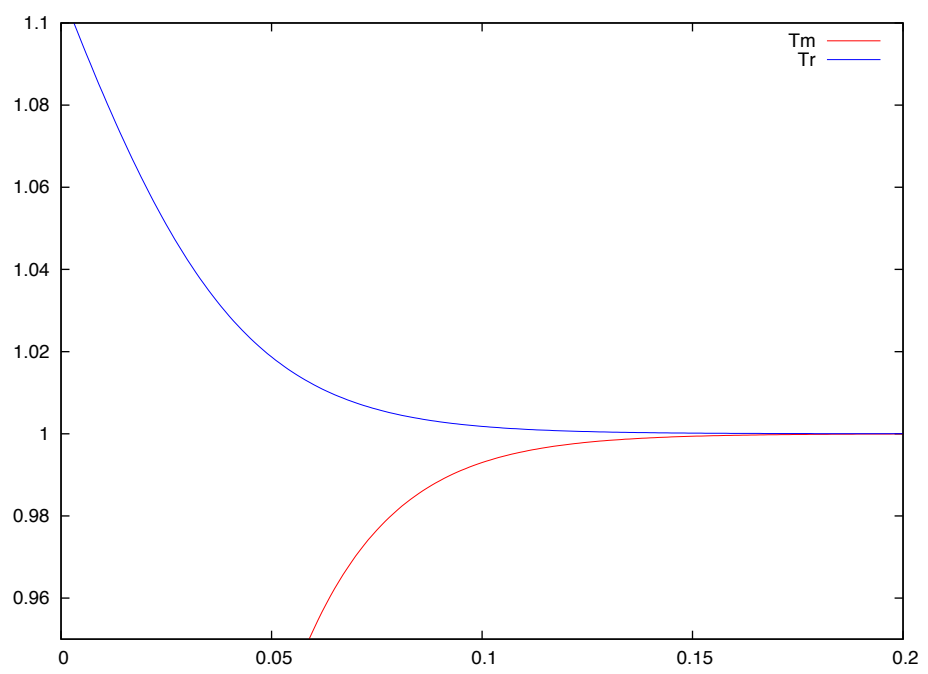

Figure 5: Matter and radiation temperature using modified IMC for infinite medium test problem with $T_{r}>T_{m}$ initially.

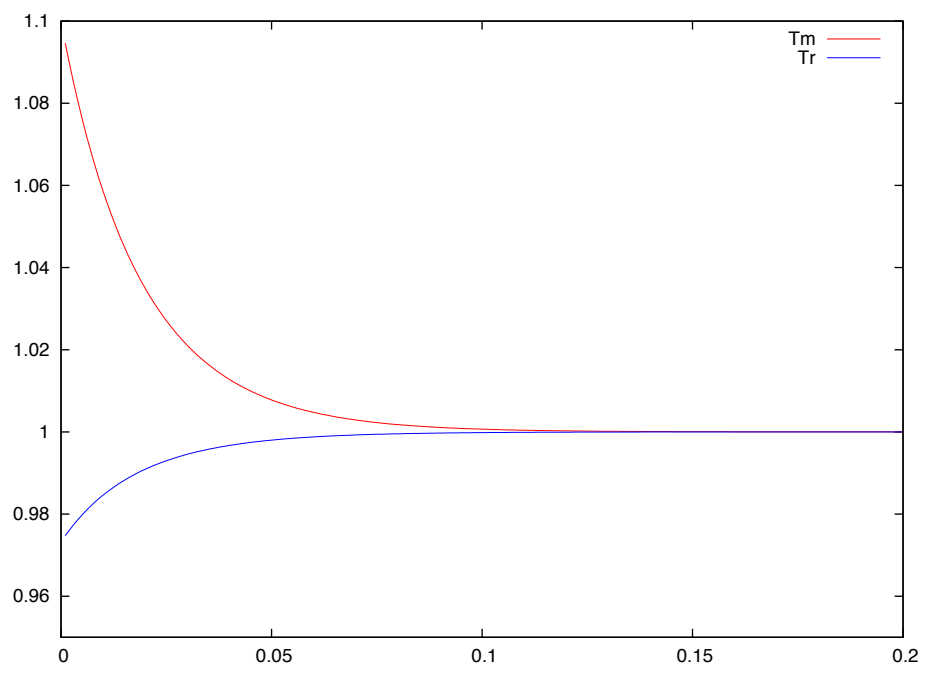

Figure 6: Matter and radiation temperature using modified IMC for infinite medium test problem with $T_{m}>T_{r}$ initially. 


\section{The student project}

The IMC results shown in Figs. 1, 2 and 3 were generated by an IMC code written in c++. I have written a small python code implementing the modified IMC algorithm for an infinite medium problem. This is how the results shown in Figs. 4, 5 and 6 were generated. I want you to take the python code and, using that as a guide, put the modified IMC algorithm into the c++ code.

You will have to add a flag to the photon class in the $\mathrm{c}++$ code that will mark the photon as either a census photon or a source photon. Census photons will be advanced by the same c++ code that they use now. The source photons will be treated by the new code you write. The new code will be very similar in structure to the already-existing IMC code. The python routines have almost the same names as the corresponding $\mathrm{c}++$ functions (for example, dBoundary calculated the distance to the boundary, advanceBoundary takes the photon, calcylated the absorption, and moves it to the boundary, etc.) So this task shouldn't be too daunting.

Once the modified IMC algorithm is coded into the IMC code, you can make sure that it reproduces the infinite medium test results of Figs. 4, 5 and 6. Then we'll try a multi-zone problem, like a 1D Marshak wave. An example of a Marshak wave problem is the one by Su and Olson, JQSRT 56 (1996), p. 337. IMC results for this test problem are shown in Fig. 7.

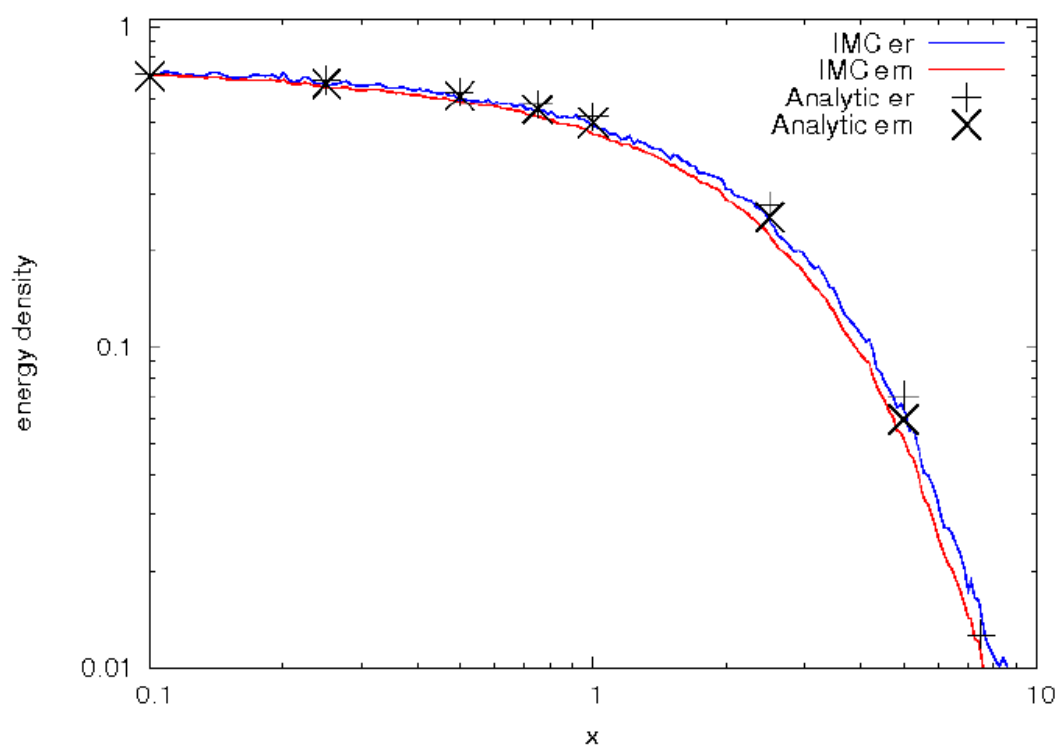

Figure 7: Matter and radiation energy for Su-Olson Marshak wave using IMC, compared to analytic answers

The Su-Olson Marshak wave has analytic answers, but only because it has a prescribed unphysical heat capacity and a fixed opacity. I think we want to test the modified algorithm on a 
range of opacities, so we will use a different but similar problem. A more reasonable reasonable problem would be 10 or 20 zones spanning $x=[0,1]$, with $T_{m}=T_{r}=0.01$ and a $T=1$ face source on it. We can change $\sigma$ and $\Delta t$ and see how the noise in the simulation varies.

After that, you make a talk, give it at Livermore. Then you can submit a write-up to an ANS conference and give the talk again. Possibly we will be able to take the results and this document and turn it into a paper for Journal of Computational Physics. I think the results in this document are good enough for an ANS talk, but I think we need to see that the modified IMC method works on a more realistic problem to make it worth a journal article. 


\section{ACKNOWLEDGMENTS}

This work performed under the auspices of the U.S. Department of Energy by Lawrence Livermore National Laboratory under Contract DE-AC52-07NA27344. 